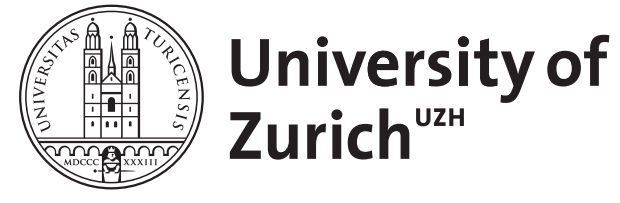

\title{
Nachsorge Schenkelhalsfraktur
}

Tandjung, R ; Rosemann, T

\begin{abstract}
Hip fractures are common and associated with high mortality and morbidity. Patients with hip fractures have a high risk of refractures. Osteoporosis is the underlying cause. A therapy with bisphosphonates is indicated without the need of further diagnostic testing with a bone marrow densitometry.
\end{abstract}

DOI: https://doi.org/10.1024/1661-8157/a000614

Other titles: Post hip fracture care

Posted at the Zurich Open Repository and Archive, University of Zurich ZORA URL: https://doi.org/10.5167/uzh-50551

Journal Article

Accepted Version

Originally published at:

Tandjung, R; Rosemann, T (2011). Nachsorge Schenkelhalsfraktur. Praxis, 100(15):917-921.

DOI: https://doi.org/10.1024/1661-8157/a000614 


\author{
Mini-Review \\ Herkunft der Arbeit \\ 1 Institut für Hausarztmedizin und Versorgungsforschung der Universität Zürich \\ 2 Klinik und Poliklinik für Innere Medizin, Universitätsspital Zürich
}

Autoren: Ryan Tandjung ${ }^{1,2}$, Thomas Rosemann ${ }^{1}$

Haupttitel: Nachsorge Schenkelhalsfraktur

Englischer Titel: Post hip fracture care

\title{
Schlüsselwörter
}

Dt. Schenkelhalsfraktur Nachsorge Bisphosphonate Hausarzt

Engl. Hip fracture Primary care bisphosphonate

\section{Zusammenfassung}

Dt. Schenkelhalsfrakturen sind häufig und gehen mit einer erhöhten Mortalität und

Morbidität einher. Nach einer Schenkelhalsfraktur besteht ein stark erhöhtes

Refrakturrisiko. Die zugrundeliegende Osteoporose sollte medikamentös mit einem

Bisphosphonat therapiert werden, eine weiterführende Diagnostik wie eine

Osteodensitometrie ist in der Regel nicht indiziert.

Engl. Hip fractures are common and associated with high mortality and morbidity. Patients with hip fractures have a high risk of refractures. Osteoporosis is the underlying cause. A therapy with bisphosphonates is indicated without the need of further diagnostic testing with a bone marrow densitometry.

\section{Haupttext}

\section{Einleitung - Epidemiologie und Risikofaktoren}

Schenkelhalsfrakturen sind häufig und mit einer hohen Morbidität und Mortalität assoziiert. Sie werden zu den osteoporotischen Frakturen gezählt und vor allem die Altersgruppe um 75 und älter sind am stärksten davon betroffen. ${ }^{1}$ Gemäss einer Studie aus Bern ist in der Schweiz die Wahrscheinlichkeit der 50-jährigen Männer bei 20.2\% und jene der Frauen $51.3 \%$ sich in ihrem restlichen Leben eine osteoporotische Fraktur zuzuziehen, wobei die Schenkelhalsfraktur mit 7\% bei Männern und $22.6 \%$ bei Frauen projiziert wird. ${ }^{2}$

Die hauptsächlich verantwortlichen Risikofaktoren sind die Osteoporose und die Sturzgefährdung. ${ }^{3}$ Wobei Faktoren wie die Malnutrition im Sinne von Untergewicht und Sehstörungen der Tiefenwahrnehmung und des Kontrastes, sowie die Medikation z.B. 
Benzodiazepinen, Antiepileptika oder Antihypertensiva mitverantwortlich sind. ${ }^{4}$ Morbus Parkinson und die Demenz $z^{5}$ wurden ebenso als Risikofaktoren, insbesondere für eine wiederholte Schenkelhalsfraktur, identifiziert. Bei Parkinson besteht eine erhöhte haltungsbedingte Sturzgefahr, bei der Demenz wird diese auf die kognitiven Einschränkung und die Tendenz herumzuwandeln zurückgeführt. Eine vorangegangene osteoporotische Fraktur bedeutet gegenüber der Normalbevölkerung ein um viermal erhöhtes Risiko eine erneute osteoporotische Fraktur zu erleiden. ${ }^{6}$

Auf die Prävention eines Sturzes und ein Screening nach Osteoporose kann in diesem Artikel nicht eingegangen werden. Wir verweisen auf aktuelle Literatur ${ }^{7}$ und die Richtlinien der Schweizerischen Vereinigung gegen Osteoporose. ${ }^{8}$

Die Schenkelhalsfraktur geht mit einer stark erhöhten perioperativen Mortalität einher. Im Zeitraum der ersten drei Monate nach Schenkelhalsfraktur besteht ein im Vergleich zur gleichen Altersgruppe 5-8-fach erhöhtes Mortalitätsrisiko. ${ }^{9}$ Die relative Risikoerhöhung nimmt im Verlauf ab, bleibt aber auch über einen weiteren Zeitraum von 10 Jahren erhalten. Ursachen für die perioperative Mortalitätserhöhung sind die mit der Operation und dem polymorbiden Patientengut assoziierten Risiken wie Infektkomplikationen, thromboembolische Ereignisse, Herzinsuffizienz oder andere pulmonale Komplikationen. Auch weitere Comorbiditäten wie Demenz, COPD, psychiatrische, neurologische und kardiovaskuläre Erkrankungen, die teilweise auch mit erhöhten Sturzrisiken einhergehen, stellen wahrscheinlich mitverantwortliche Faktoren für die erhöhte Mortalität dar.

Eine Basler Studie zeigte, dass ein Jahr nach einer Schenkelhalsfraktur - bei einem Durchschnittsalter von 84 Jahren - 22\% der Patienten verstorben sind, wobei hier die Mortalität im Wesentlichen auch von der Funktionalität der Patienten vor der Operation abhing. So zeigte sich, dass von jenen Patienten, die zum Zeitpunkt der Fraktur bereits in einem Pflegeheim (oder in einem Spital) untergebracht waren, 44\% verstarben, im Vergleich zu den $12 \%$ in der Gruppe die zuvor zu Hause wohnhaft waren. ${ }^{10}$

Die Morbiditätslast zeigt sich im Einfluss auf die Selbständigkeit im Alltag und in der Pflegebedürftigkeit. Die Basler Studie hat hier aufgezeigt, dass von den ursprünglich 158 Patienten, die zuvor zu Hause gewohnt hatten, 131 (83\%) wieder nach Hause kehrten. Die Anzahl jener Personen, die ohne jegliche Hilfe das Alltagsleben meistern konnten, sank von 69\% auf 39\%. Vor der Fraktur berichteten 126 Personen (80\%) eine Gehfähigkeit ohne zusätzliche Unterstützung, diese Anzahl sank auf 90 (57\%) ein Jahr nach dem stattgehabten Unfall. ${ }^{10}$ 
Mit zunehmendem Alter nimmt die Häufigkeit der Frakturen und die damit assoziierte Mortalität $z u^{1}$, dies einerseits weil die Knochendichte abnimmt und andererseits weil durch Gangunsicherheit, medikamentöse Nebenwirkungen und Comorbiditäten die Sturzgefahr zunimmt. Angesichts der zunehmend älteren Bevölkerung ist mit einer Zunahme von osteoporotischen Frakturen zu rechnen.

\section{Therapie}

Die Osteoporose ist definiert als Erkrankung mit erniedrigter Knochenmasse und Verlust der Mikrostruktur des Knochengewebes, was zu erhöhter Knochenbrüchigkeit und einem damit erhöhten Frakturrisiko führt. ${ }^{11}$ Die Schenkelhalsfraktur ihrerseits ist also Ausdruck der zugrundeliegenden Osteoporose und bedarf entsprechender diagnostischer und therapeutischer Massnahmen. Die operative Therapie und die perioperativen Begleitmassnahmen können aufgrund der Komplexität des Themas in diesem Artikel nicht behandelt werden.

Im Jahre 2007 wurden in der Schweiz etwas mehr als 20'000 Patienten mit einer osteoporotischen Fraktur hospitalisiert, wobei rund die Hälfe auf Schenkelhalsfraktur zurückzuführen ist. Für die Hospitalisation lässt sich eine durchschnittliche Spitalaufenthaltsdauer von knapp elf Tagen aufzeigen, dies ist zwar eine deutliche Abnahme gegenüber vergleichbaren Zahlen in den Jahren zuvor. Die Kosten jedoch waren aufgrund der steigenden Spitalkosten insgesamt trotz kürzerer Hospitalisation ansteigend. Kostenschätzungen im 2007 belaufen sich für die Frauen auf 171 Mio. und für Männer auf 58.2 Mio. Franken. ${ }^{12}$

\section{"Osteoporosis is a silent disease, until it is complicated by fractures" 13}

Die Schenkelhalsfraktur darf nicht als isoliertes Ereignis angesehen werden. Patienten, die eine osteoporotische Fraktur erlitten haben, haben ein erhöhtes Risiko erneute eine Fraktur zu erleiden, ${ }^{14}$ eine Untersuchung zeigte auf, dass $45 \%$ der Patienten, die eine Fraktur erlitten, eine weitere Fraktur erleiden werden. Angesichts der mit der Fraktur assoziierten hohen Mortalität, sowie des älteren Patientengutes konnte nach entsprechender Korrektur gezeigt werden, dass diese Refrakturrate der Überlebenden in den 10 Jahren nach der Fraktur auf 85\% ansteigt, d.h. die Chance nach der Schenkelhalsfraktur 10 Jahre und ohne erneute Fraktur zu überleben liegt bei nur $15 \%{ }^{1}$

Angesichts der hohen Rate an erneuten Frakturen ist eine Therapie der zugrundeliegenden Osteoporose indiziert. Neben Vitamin D und Calcium, wurde vor allem 
auch die Rolle der Bisphosphonate untersucht. Diese Präparate sind teuer, zeigten allerdings in Kosten-Nutzen-Untersuchungen insbesondere unter Berücksichtigung von (Re-)Frakturen und den damit verbundenen Hospitalisationen und Folgekosten eine gute Kosteneffizienz ${ }^{15,16}$. In einer doppel-blinden, randomisierten, Placebo-Kontrollierten Studie konnte ein positiver Einfluss der Bisphosphonate auf das Refrakturrisiko und die Mortalität gezeigt werden. ${ }^{17}$ Die Intervention führte zu einer Reduktion der Refrakturrate um 35\% und einer Mortalitätsreduktion um 28\%. Eine Untersuchung in den USA im Rahmen eines in der HMO „Kaiser Permanente“ initiierten Programms zeigte nach Intensivierung der Osteoporosetherapie eine Reduktion der Frakturen um 37.2\%. ${ }^{18}$ Schliesslich kann auch mit Verhinderung von Frakturen auch der Verlust von Selbständigkeit und eine Pflegebedürftigkeit vermindert werden. ${ }^{10,19}$

Diese Aspekte haben sich in den Leitlinien niedergeschlagen. Das Risiko, erneut eine Fraktur zu erleiden, ist unabhängig von der gemessenen Osteodensitometrie, erhöht ${ }^{14}$. Die Schweizerische Vereinigung gegen Osteoporose (SVGO) empfiehlt in ihren Richtlinien eine Therapie mit Bisphosphonaten, ohne grundsätzlich Zeit und Ressourcen für eine Osteodensitometrie aufzuwenden, falls ein Patient eine osteoporotische Fraktur erlitten hat. ${ }^{8}$

Einige internationale Richtlinien (USA, Europäische Richtlinien, Deutschland ${ }^{3,13,20}$ ) kommen zu ähnlichen Empfehlungen, wobei hingegen weitere Richtlinien (Canada, Grossbritannien bei Männern ${ }^{21,22}$ ) vor einer Therapie die Durchführung einer Osteodensitometrie empfehlen und in begründeten Fällen auf die vorangehende Diagnostik verzichten, zudem beziehen sich einige Richtlinien auf unterschiedliche Altersgruppen oder die europäische Richtlinie nur auf postmenopausalen Frauen. ${ }^{3}$

Unabhängig der leicht unterschiedlichen Richtlinien, die in den letzten zehn Jahren implementiert wurden, zeigt die Realität eine sehr niedrige Rate an Patienten, die eine empfohlene Diagnostik oder Therapie erhalten hatten. ${ }^{23-28}$. Unterschiedliche Ursache dafür wurden postuliert und untersucht, so z.B. die fehlende Information der Patienten über den Zusammenhang der Fraktur und einer zugrundeliegenden Osteoporose ${ }^{29}$ oder die Frage nach Zuständigkeit der mittelfristig angesetzten Therapie, schliesslich ist der Unfallchirurge / Orthopäde jener Arzt, der den Patienten als erstes sieht. ${ }^{30}$ In dieser Studie, die in den USA durchgeführt wurde, sind sowohl die involvierten Grundversorger als auch die behandelnden Orthopäden zum Schluss gekommen sind, dass der Hausarzt das medikamentöse Management der Osteoporose in der Hand halten sollte. 
Unterschiedliche Interventionen, um die Therapierate zu erhöhen, wurden untersucht. So wurden Fallmanager ${ }^{31}$ eingesetzt oder gezielt Patienten stärker über ihre Erkrankung und über die Notwendigkeit der Therapie informiert ${ }^{32,33}$ oder es erfolgte eine gezielte Information an die nachbehandelnden Ärzte ${ }^{34,35}$. Eine Übersichtsarbeit von Little et al. über verschiedene Interventionen konnte bei allen Interventionen eine Erhöhung der Guideline-Adhärenz zeigen, die Autoren geben allerdings auch zu bedenken, dass bei der geringen initialen Guideline-Adhärenz jegliche Intervention eine Therapieerhöhung erbringen kann und bei allen Interventionen die Adhärenz noch einmal deutlich verbesserten werden könnte. ${ }^{36}$

In Genf wurde ein spezifischer Patientenweg für osteoporotische Frakturen entwickelt. Die Anmeldung erfolgte durch den behandelnden Arzt. Der Patientenweg bestand im ersten Schritt aus der Vervollständigung der Anamnese (aktuelle und allfällig vorhergehende Frakturen), sowie der Überprüfung des Calciumhaushaltes und der Ernährungssituation. In einem zweiten Schritt erfolgte die Durchführung einer Osteodensitometrie, wobei diese nur dann durchgeführt wurde, wenn nach klinischem Ermessen eine Therapieänderung aus den Resultaten folgen würde. In einem dritten Schritt wurden individuell angepasste Empfehlung erstellt und diese an den behandelnden Orthopäden und Hausarzt versandt. ${ }^{37}$ In dieser Studie wurde aufgrund der Altersverteilung (viele Patienten gehörten eine sehr betagte Gruppe an) am Ende nur rund in 1/3 der Fälle der Beginn einer spezifischen Anti-Osteoporosetherapie empfohlen.

\section{Zeitpunkt der Bisphosphonatgabe}

Das Risiko einer erneuten Fraktur ist zeitlich abhängig von der erlittenen Fraktur. Unmittelbar nach der (ersten) Fraktur ist das Risiko am stärksten erhöht ${ }^{38-40}$, was die Notwendigkeit einer frühen Intervention aufzeigt. Würde eine Bisphosphonattherapie unmittelbar an die Fraktur anschliessen, könnte aufgrund der lokalen osteoplastischen Aktivität um den Frakturbereich, ein unzureichender Effekt auf die restlichen Areale zu erwarten sein. Eine doppelblinde, Placebo-Kontrollierte und randomisierte Studie ${ }^{41}$ untersuchte deshalb den idealen Zeitpunkt des Beginns einer jährlichen intravenösen Bisphosphonattherapie (Zoledronsäure, Aclasta®) vs. Placebo. Dabei wurde initial und im Verlauf eine Knochendichtemessung durchgeführt und die Refrakturraten wurden beobachtet (primärer Endpunkt). Die Analyse ergab eine verminderte medikamentöse Effektivität, wenn Bisphosphonate innerhalb von zwei Wochen nach der Fraktur gegeben wurden. Den besten Effekt auf die Refrakturrate ergab der Medikationszeitpunkt 2-4 Wochen nach der Fraktur. 
Eine Schenkelhalsfraktur ist als „Red flag“ einer zugrundeliegenden Osteoporose zu sehen und bedarf gegebenenfalls weiterführender Abklärung und die Einleitung einer Osteoporosetherapie mit Vitamin D, Calcium und einem Bisphosphonat.

\section{Key Points}

- Eine Schenkelhalsfraktur ist mit hoher Morbidität und Mortalität verbunden und eine frühzeitige Therapie zur Verhinderung einer erneuten Fraktur ist angezeigt.

- Patienten mit erlittenen Schenkelhalsfrakturen haben ein hohes Risiko erneute Frakturen zu erleiden, die zugrundeliegende Osteoporose sollte medikamentös mit einem Bisphosphonat therapiert werden.

- Auf eine Osteodensitometrie vor Therapiebeginn kann in der Regel nach einer Schenkelhalsfraktur verzichtet werden.

\section{Lernfragen}

1. Wie viele der heute 50 -jährigen Frauen werden in ihrem restlichen Leben eine osteoporotische Fraktur erleiden?
a. Weniger als $10 \%$
b. Ein Drittel
c. Etwa die Hälfe
d. Fast alle

2. Wie hoch ist in etwa die Wahrscheinlichkeit nach einer erlittenen Schenkelhalsfraktur die nächsten 10 Jahre ohne weitere osteoporotische Fraktur zu überleben?
a. $<5 \%$
b. $15 \%$
c. $40 \%$
d. $70 \%$

3. Eine 70-jährige Patientin kommt eine Woche nach erlittener Schenkelhalsfraktur in die hausärztliche Nachkontrolle, was ist in Hinblick auf die Osteoporose Ihr weiteres Vorgehen?
a. Ich melde eine Osteodensitometrie an
b. Ich beginne heute mit einer Bisphosphonattherapie
c. Osteoporose spielt hier keine Rolle 


\title{
d. Ich beginne mit Vitamin D, Calcium und im Verlauf des kommenden Monats mit Bisphosphonaten
}

Richtige Antworten: 1 c 2 b 3 d

\author{
Korrespondenzadresse \\ Dr. med. Ryan Tandjung \\ Institut für Hausarztmedizin und Versorgungsforschung \\ Klinik und Poliklinik für Innere Medizin \\ UniversitätsSpital Zürich \\ 8091 Zürich \\ ryan.tandjung@usz.ch
}

\section{Referenzen}

1. von Friesendorff M, Besjakov J, Akesson K. Long-term survival and fracture risk after hip fracture: a 22-year follow-up in women. J Bone Miner Res 2008;23:1832-41.

2. Lippuner K, Johansson H, Kanis JA, Rizzoli R. Remaining lifetime and absolute 10-year probabilities of osteoporotic fracture in Swiss men and women. Osteoporos Int 2009;20:1131-40.

3. Kanis JA, Burlet N, Cooper C, et al. European guidance for the diagnosis and management of osteoporosis in postmenopausal women. Osteoporos Int 2008;19:399-428.

4. Cummings SR, Nevitt MC, Browner WS, et al. Risk factors for hip fracture in white women. Study of Osteoporotic Fractures Research Group. N Engl J Med 1995;332:767-73.

5. Yamanashi A, Yamazaki K, Kanamori M, et al. Assessment of risk factors for second hip fractures in Japanese elderly. Osteoporos Int 2005;16:1239-46.

6. Chapurlat RD, Bauer DC, Nevitt M, Stone K, Cummings SR. Incidence and risk factors for a second hip fracture in elderly women. The Study of Osteoporotic Fractures. Osteoporos Int 2003;14:130-6.

7. Michael YL, Whitlock EP, Lin JS, Fu R, O'Connor EA, Gold R. Primary Care-Relevant Interventions to Prevent Falling in Older Adults: A Systematic Evidence Review for the U.S. Preventive Services Task Force. Annals of Internal Medicine 2010;153:815-25.

8. SVGO SVgdO. Osteoporose: Prävention - Diagnostik - Behandlung Empfehlungen 2010. online version on wwwsvgoch 2010.

9. Haentjens P, Magaziner J, Colon-Emeric CS, et al. Meta-analysis: excess mortality after hip fracture among older women and men. Ann Intern Med 2010;152:380-90.

10. Pretto M, Spirig R, Kaelin R, Muri-John V, Kressig RW, Suhm N. Outcomes of elderly hip fracture patients in the Swiss healthcare system: A survey prior to the implementation of DRGs and prior to the implementation ofa Geriatric Fracture Centre. Swiss Med Wkly 2010;140:w13086.

11. Kanis JA, Melton LJ, 3rd, Christiansen C, Johnston CC, Khaltaev N. The diagnosis of osteoporosis. J Bone Miner Res 1994;9:1137-41.

12. Lippuner K, Popp AW, Schwab P, et al. Fracture hospitalizations between years 2000 and 2007 in Switzerland: a trend analysis. Osteoporos Int 2010.

13. NOF NOF. Clinician's Guide to Prevention and Treatment of Osteoporosis. online version on wwwnoforg 2010.

14. Klotzbuecher CM, Ross PD, Landsman PB, Abbott TA, 3rd, Berger M. Patients with prior fractures have an increased risk of future fractures: a summary of the literature and statistical synthesis. J Bone Miner Res 2000;15:721-39.

15. Dell R, Greene D. Is osteoporosis disease management cost effective? Curr Osteoporos Rep 2010;8:49-55.

16. Newman ED, Ayoub WT, Starkey RH, Diehl JM, Wood GC. Osteoporosis disease management in a rural health care population: hip fracture reduction and reduced costs in postmenopausal women after 5 years. Osteoporos Int 2003;14:146-51.

17. Lyles KW, Colon-Emeric CS, Magaziner JS, et al. Zoledronic acid and clinical fractures and mortality after hip fracture. N Engl J Med 2007;357:1799-809. 
18. Dell R, Greene D, Schelkun SR, Williams K. Osteoporosis disease management: the role of the orthopaedic surgeon. J Bone Joint Surg Am 2008;90 Suppl 4:188-94.

19. Cooper $C$. The crippling consequences of fractures and their impact on quality of life. Am J Med 1997;103:12S-7S; discussion 7S-9S.

20. DVO DOeV. DVO-Leitlinie 2009 zur Prophylaxe, Diagnostik und Therapie der Osteoporose bei Erwachsenen. online version on wwwdv-osteologiede 2009.

21. Compston J, Cooper A, Cooper C, et al. Guidelines for the diagnosis and management of osteoporosis in postmenopausal women and men from the age of 50 years in the UK. Maturitas 2009;62:105-8.

22. Papaioannou A, Morin S, Cheung AM, et al. 2010 clinical practice guidelines for the diagnosis and management of osteoporosis in Canada: summary. CMAJ 2010;182:1864-73.

23. Elliot-Gibson V, Bogoch ER, Jamal SA, Beaton DE. Practice patterns in the diagnosis and treatment of osteoporosis after a fragility fracture: a systematic review. Osteoporos Int 2004;15:767-78.

24. Jennings LA, Auerbach AD, Maselli J, Pekow PS, Lindenauer PK, Lee SJ. Missed opportunities for osteoporosis treatment in patients hospitalized for hip fracture. J Am Geriatr Soc 2010;58:650-7.

25. Harrington JT, Broy SB, Derosa AM, Licata AA, Shewmon DA. Hip fracture patients are not treated for osteoporosis: a call to action. Arthritis Rheum 2002;47:651-4.

26. Rabenda V, Vanoverloop J, Fabri V, et al. Low incidence of anti-osteoporosis treatment after hip fracture. J Bone Joint Surg Am 2008;90:2142-8.

27. Suhm N, Lamy O, Lippuner K. Management of fragility fractures in Switzerland: results of a nationwide survey. Swiss Med Wkly 2008;138:674-83.

28. Feldstein AC, Nichols GA, Elmer PJ, Smith DH, Aickin M, Herson M. Older women with fractures: patients falling through the cracks of guideline-recommended osteoporosis screening and treatment. J Bone Joint Surg Am 2003;85-A:2294-302.

29. Giangregorio L, Papaioannou A, Thabane L, et al. Do patients perceive a link between a fragility fracture and osteoporosis? BMC Musculoskelet Disord 2008;9:38.

30. Simonelli C, Killeen K, Mehle S, Swanson L. Barriers to osteoporosis identification and treatment among primary care physicians and orthopedic surgeons. Mayo Clin Proc 2002;77:334-8.

31. Majumdar SR, Beaupre LA, Harley $\mathrm{CH}$, et al. Use of a case manager to improve osteoporosis treatment after hip fracture: results of a randomized controlled trial. Arch Intern Med 2007;167:2110-5.

32. Gardner MJ, Brophy RH, Demetrakopoulos D, et al. Interventions to improve osteoporosis treatment following hip fracture. A prospective, randomized trial. J Bone Joint Surg Am 2005;87:3-7.

33. Majumdar SR, Johnson JA, McAlister FA, et al. Multifaceted intervention to improve diagnosis and treatment of osteoporosis in patients with recent wrist fracture: a randomized controlled trial. CMAJ 2008;178:569-75.

34. Solomon DH, Polinski JM, Stedman M, et al. Improving care of patients at-risk for osteoporosis: a randomized controlled trial. J Gen Intern Med 2007;22:362-7.

35. Feldstein A, Elmer PJ, Smith DH, et al. Electronic medical record reminder improves osteoporosis management after a fracture: a randomized, controlled trial. J Am Geriatr Soc 2006;54:450-7.

36. Little EA, Eccles MP. A systematic review of the effectiveness of interventions to improve postfracture investigation and management of patients at risk of osteoporosis. Implement Sci 2010;5:80.

37. Chevalley T, Hoffmeyer P, Bonjour JP, Rizzoli R. An osteoporosis clinical pathway for the medical management of patients with low-trauma fracture. Osteoporos Int 2002;13:450-5.

38. Ryg J, Rejnmark L, Overgaard S, Brixen K, Vestergaard P. Hip fracture patients at risk of second hip fracture: a nationwide population-based cohort study of 169,145 cases during 1977-2001. J Bone Miner Res 2009;24:1299-307.

39. van Geel TA, van Helden S, Geusens PP, Winkens B, Dinant GJ. Clinical subsequent fractures cluster in time after first fractures. Ann Rheum Dis 2009;68:99-102.

40. Chen JS, Cameron ID, Simpson JM, et al. Low-trauma fractures indicate increased risk of hip fracture in frail older people. J Bone Miner Res 2011;26:428-33.

41. Eriksen EF, Lyles KW, Colon-Emeric CS, et al. Antifracture efficacy and reduction of mortality in relation to timing of the first dose of zoledronic acid after hip fracture. J Bone Miner Res 2009;24:1308-13. 